\title{
Choroidal vascularity index as an indicator of vascular status of choroid, in eyes with nanophthalmos
}

\author{
Funda Ebru Aksoy ${ }^{1} \cdot$ Cigdem Altan $^{1} \cdot$ Cem Kesim $\mathbb{1}^{2} \cdot$ Ali Demircan $^{1} \cdot$ Ugur Tunç $\mathbb{D}^{1} \cdot$ Gokhan Demir $^{3}{ }^{3}$. \\ Muhittin Taskapılı ${ }^{1}$
}

Received: 26 January 2020 / Revised: 30 April 2020 / Accepted: 6 May 2020 / Published online: 15 May 2020

(c) The Author(s), under exclusive licence to The Royal College of Ophthalmologists 2020

\begin{abstract}
Purpose To investigate choroidal vascular index (CVI) in eyes with nanophthalmos (NO) with the use of optical coherence tomography (OCT).

Methods Macular enhanced depth imaging OCT scans of 25 eyes of 25 patients with NO and age-gender-matched 25 eyes of 25 control subjects were analysed. Images were binarized using the ImageJ software, and total choroid area (TCA), luminal area (LA) and stromal area (SA) were acquired. The main outcome measure was CVI, defined as the ratio of LA to TCA.

Results Twenty-five eyes of 25 patients with NO and age-gender-matched control subjects were enrolled. The mean TCA, SA and LA were found to be significantly higher in patients with $\mathrm{NO}\left(2.51 \pm 0.44\right.$ vs. $1.91 \pm 0.35 \mathrm{~mm}^{2}, P<0.001 ; 0.86 \pm$ 0.17 vs. $0.63 \pm 0.13 \mathrm{~mm}^{2}, P<0.001$; and $1.65 \pm 0.29$ vs. $1.27 \pm 0.23 \mathrm{~mm}^{2}, P<0.000$, respectively). On the contrary, CVI did not significantly differ between the two groups $(65.72,67.68, P=0.099)$.

Conclusion As a novel OCT-based marker, CVI could be used to assess vascular status of the choroid in eyes with NO and can provide better understanding of the pathogenesis of this disease.
\end{abstract}

\section{Introduction}

Nanophthalmos (NO) is a subtype of microphthalmos which includes reduction in size of the eye in the absence of any other congenital ocular anomalies [1]. Clinically patients with NO have shallow anterior chamber, high hyperopia, axial length of $<21 \mathrm{~mm}[2-5]$ and they have thicker choroid and sclera compared to the normal eyes [6].

NO is believed to result from arrested growth of the eye during the embryonic period and they usually develop posterior segment complications including relapsingremitting uveal effusion, macular hypoplasia, cysts and

Funda Ebru Aksoy

fundaonmez@sakarya.edu.tr

1 University of Health Science, Istanbul Beyoglu Eye Training and Research Hospital, Istanbul, Turkey

2 Koc University, Department of Ophthalmology, Istanbul, Turkey

3 University of Health Science, Fatih Sultan Mehmet Training and Research Hospital, Istanbul, Turkey striae, disc pallor, pseudopapilloedema and hyperpigmentation of the retinal pigment epithelium (RPE) [7].

With the development of optical coherence tomography (OCT) technology, such as enhanced depth imaging (EDI) and swept-source OCT, the visualization of choroidal structures along with a more accurate calculation of multiple quantitative parameters is now possible. Recently, the measurement of choroidal thickness (CT) has been frequently used in several diseases; however, it might be influenced by some biological variables, such as axial length, refractive error, intraocular pressure, and systolic blood pressure and it does not give information separately on the changes of the vascular and stromal choroidal components [8]. Thus, to overcome these problems, image binarization techniques were developed and applied on EDI-OCT scans, which allows us to analyse and quantify the choroidal vascular and stromal structures, separately $[8,9]$. Choroidal vascularity index (CVI) which corresponds to the ratio between choroidal luminal area (LA) to total choroidal area (TCA) has been defined as a new tool to assess choroidal vascular status [8-12]. In this study, we aimed to evaluate for the first time CVI in patients with NO and to compare with healthy controls. 


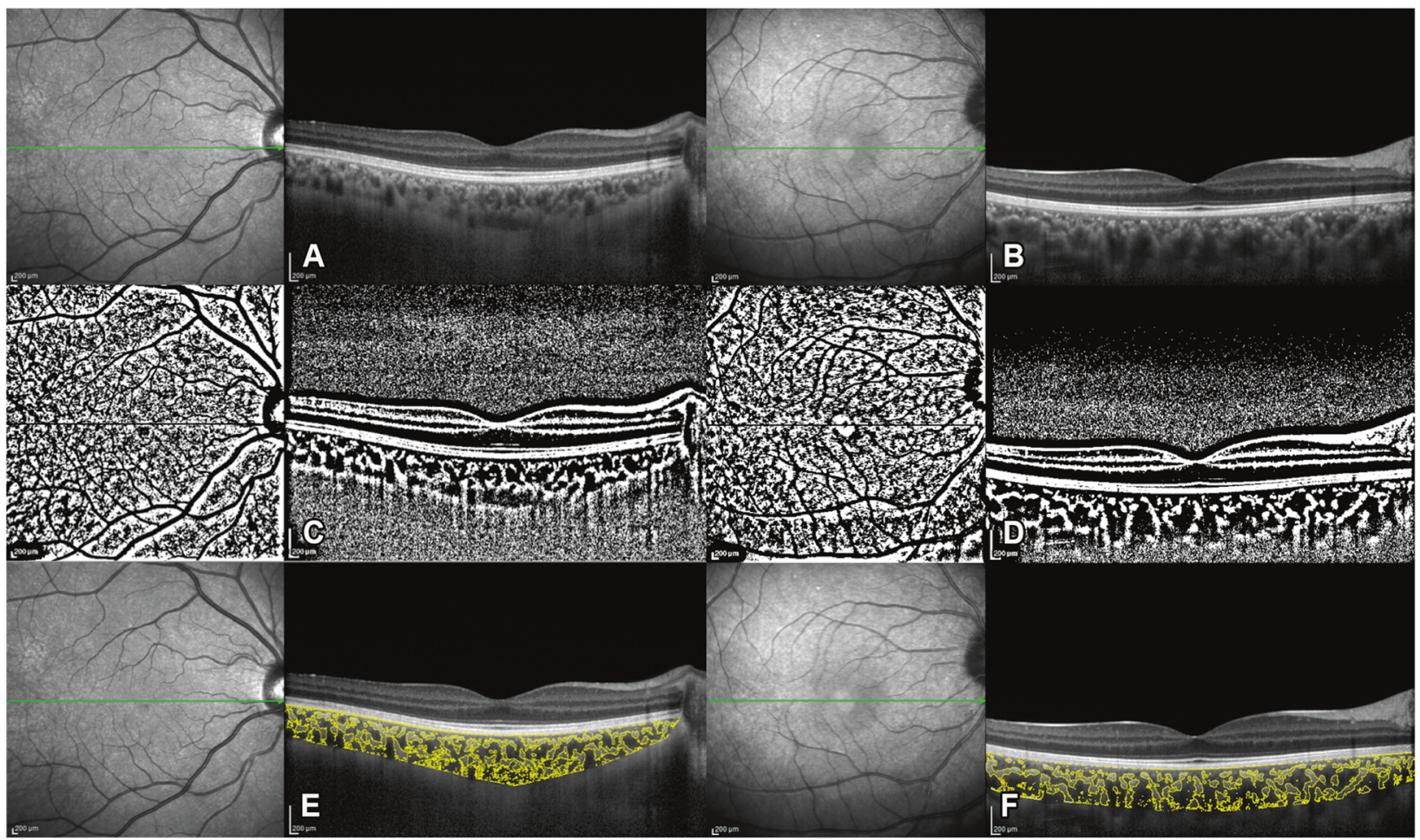

Fig. 1 Measurement technique of choroidal vascularity index (CVI) in a control subject and in a patient with nanophthalmos. a, b The enhanced depth optical coherence tomography (EDI-OCT) images of the subjects. c, d The EDI-OCT scans were binarized to

\section{Methods}

Patients with NO who have been followed up at our clinic were enrolled in this study. The diagnosis of nanopthalmos was defined as a shorter than average $\mathrm{AL}(\leq 20 \mathrm{~mm})$, a shallow anterior chamber and hyperopia ( +3.5 dioptres). Control group consisted of age-gender-matched healthy emmetropic individuals.

Exclusion criteria were trauma, intraocular surgery, systemic diseases, systemic malformations, systemic or topical medications, optic neuropathy, any retinal pathology other than NO-related retinal findings, such as pseudopapilloedema, papillomacular folds and leopard spots.

Ophthalmological evaluation, was consisted of the measurement of best corrected visual acuity, intraocular pressure measurement with a Goldmann applanation tonometer, slit-lamp biomicroscopical examination of anterior segment and dilated fundus examination was performed for all the participants. Measurement of AL was performed with IOL Master optical biometry (IOL-Master, V.918471, model 1322-734; Carl Zeiss Meditec, La Jolla, CA, USA).

Imaging of the choroid was performed after pupil dilation with tropicamide 1\%, using EDI mode of Spectralis OCT-imaging device (Heidelberg Engineering, Germany). Imaging protocol comprised of 49 horizontal $9 \mathrm{~mm}$ raster Bscans centred at the fovea per volume scan of $30^{\circ} \times 30^{\circ}$. determine the total choroidal area (TCA). e, f The colour threshold tool was used to select the dark pixels, representing the luminal areas (LA). Calculation of CVI was performed by dividing LA to TCA.

Sub-foveal choroidal thickness was measured manually at the fovea using the calliper tool in the software, as the vertical distance between the hyper reflective line of Bruch's membrane and the hyper-reflective line of the chorio-scleral interface. Only good quality scans with signal strength $\geq 7$ were used for analysis. All scans were taken between 8.30 and 11.30 a.m.

For measurement of CVI, macular SD-OCT scans were binarized by using the public domain software ImageJ 1.51s (National Institutes of Health, Bethesda, MD, USA), with a semi-automated technique previously described. The EDI-OCT image was opened with ImageJ, and the polygon tool was used to assess the region of interest (ROI) across the entire length of the OCT scan. The upper boundary of the ROI was traced along the basal margin retina pigment epithelium and the lower boundary along the choroidalscleral border in order to define the TCA. Furthermore, the image was converted to eight bits and autolocal thresholding (Niblack method) was performed to this binarized image [8]. In order to select dark pixels, representing the LA, the image was again converted into red, green, blue (RGB). The LA was calculated as the sum of dark pixel areas, and the interstitial or stromal area (SA) was computed from the subtraction of LA from TCA of the ROI; the CVI, was calculated as the ratio between LA and TCA (Fig. 1). 
Table 1 Demographic and clinical characteristics of patients with nanophthalmos (NO) and control subjects.

\begin{tabular}{lllc}
\hline Characteristic & NO eyes & Control eyes & $P$ value \\
\hline Age (years) (mean \pm SD) & $25.12 \pm 9.8$ & $27.16 \pm 10.2$ & 0.25 \\
Gender (female/male) & $13 / 12$ & $13 / 12$ & 1.0 \\
BCVA (logMar) (mean \pm SD) & $0.48 \pm 0.32$ & $0.00 \pm 0.06$ & 0.001 \\
Axial length $(m m)($ mean \pm SD) & $16.01 \pm 0.9$ & $22.9 \pm 0.7$ & $<0.001$ \\
\hline
\end{tabular}

Statistical analysis was performed with SPSS statistical software (SPSS Inc, Chicago, IL, USA). The KolmogorovSmirnov test was used to evaluate the normality of data. Independent-sample $t$ test was used to compare the mean values of normally distributed parameters and chi-square test was performed for categorical variables. the MannWhitney $U$ test was performed for non-normally distributed parameters. Pearson correlation coefficients were calculated to evaluate relationships between parameters. $P<0.05$ was considered statistically significant.

\section{Results}

Twenty-five eyes of 25 patients with $\mathrm{NO}$ and 25 eyes of 25 control subjects were included in this study. The baseline demographic and clinical characteristics of patients and control subjects are shown in Table 1 . There was no significant difference in regards of age $(P=0.250)$ and gender $(P=0.339)$ between the two groups. Mean BCVA was significantly different between two groups $(0.48 \pm 0.32 \mathrm{log}$ MAR in patients with nanophthalmus and $0.00 \pm 0.06 \mathrm{log}$ MAR in control subjects, $P=0.001$ ).

Subfoveal CT was significantly higher in patients with NO compared to control eyes $(405 \pm 80.41,300.97 \pm$ $45.21 \mu \mathrm{m}$, respectively, $P<0.001)$. The mean TCA, SA and LA were found to be significantly higher in patients with NO compared to control subjects $(2.51 \pm 0.44$ vs. $1.91 \pm$ $0.35 \mathrm{~mm}^{2}, P<0.001 ; 0.86 \pm 0.17$ vs. $0.63 \pm 0.13 \mathrm{~mm}^{2}, P<$ 0.001 ; and $1.65 \pm 0.29$ vs. $1.27 \pm 0.23 \mathrm{~mm}^{2}, P<0.000$, respectively). On the contrary, CVI did not significantly differ between the two groups $(65,72,67.68, P=0.099)$ (Table 2).

Correlation analyses revealed that there were no significant correlations between CVI and AL, BCVA or age $(P>0.05)$. The only parameter that correlates with CVI was SFCT $(r=-0.380, P=0.036)$.

\section{Discussion}

The role of choroidal vascularity was reported in the pathogenesis of various eye disorders and proposed an
Table 2 Comparison of luminal area (LA), stromal area (SA), choroidal vascular index (CVI) and subfoveal choroidal thickness (SFCT) between eyes with nanophthalmos (NO) and normal eyes.

\begin{tabular}{lccr}
\hline & $\begin{array}{l}\text { NO eyes } \\
(\text { mean } \pm \text { SD })\end{array}$ & \multicolumn{1}{c}{$\begin{array}{l}\text { Control eyes } \\
(\text { mean } \pm \text { SD })\end{array}$} & $P$ value \\
\hline LA $\left(\mathrm{mm}^{2}\right)$ & $1.65 \pm 0.29$ & $1.27 \pm 0.23$ & $<0.001$ \\
SA $\left(\mathrm{mm}^{2}\right)$ & $0.86 \pm 0.17$ & $0.63 \pm 0.13$ & $<0.001$ \\
TCA $\left(\mathrm{mm}^{2}\right)$ & $2.51 \pm 0.44$ & $1.91 \pm 0.35$ & $<0.001$ \\
CVI $(\%)$ & $65.72 \pm 3.21$ & $67.68 \pm 3.02$ & 0.099 \\
SFCT $(\mu \mathrm{m})$ & $405 \pm 80.41$ & $300.97 \pm 45.21$ & $<0.001$ \\
\hline
\end{tabular}

independent surrogate marker to assess choroidal health. This OCT-based marker was suggested to be a more stable measurement to monitor choroid compared to SFCT, which was influenced by some biological variables and demonstrated greater variability [8]. In this study, we found that in NO eyes choroidal thickness was increased but CVI was not affected, in these patients.

NO is a rare ocular abnormality in consequence of developmental arrest of the globe after closure of the embryonic fissure [13]. A previous study revealed that NO eyes had increased SFCT, even after the biometric parameters, e.g. AL were excluded. Authors suggest that the increased choroidal thickness might be the main pathology in NO [14].

Eyes with NO have abnormally thickened sclera that results with reduced choroidal vessel permeability to proteins and compression to the vortex vein [2]. In the literature, it was suggested that the pathogenesis of central serous chorioretinopathy (CSCR) and NO was similar. In CSCR, choroidal hyperpermeability, choroidal congestion and vortex vein outflow obstruction are proposed to be the cause of serous macular detachment [15]. However, Agarwal et al. reported that eyes with acute CSCR showed significantly higher CVI, LA and SFCT compared to fellow eyes and controls, without a significant change in SA [10]. On the contrary, in this study we found that CVI was not different in NO eyes and there was a proportional increase in LA and SA. Thus, we think that the pathogenesis of these two disorders are different.

It is possible that the choroidal thickening might be the main factor leading to the diminished eye volume and abnormal scleral composition in eyes with NO. Studies clearly revealed that the choroid takes an active role in emmetropization in two different ways; by modulation of its thickness to adjust the retina to the focal plane of the eye (choroidal accommodation), and by releasing of growth factors that regulate scleral proteoglycan synthesis. In addition to modulating the position of the retina, choroidal thickness may provide a mechanism for regulating the delivery of bioactive molecules released from the retina, RPE or choroid to the sclera. Because of this ability, a 
thicker choroid could inhibit the transfer of anabolic growth factors from retina and RPE to the sclera, diminish scleral matrix synthesis, and thus, inhibit the rate of ocular elongation [16]. Nevertheless, in another study, Marzani et al. revealed that the secreted molecules directly from the choroid could inhibit scleral proteoglycan synthesis and these molecules could regulate the rate of ocular elongation [17]. Presumably increased choroidal thickness may cause increased secretion of inhibitory molecules from the choroid and this factor could be the leading factor resulting with the diminished volume and abnormal scleral composition in $\mathrm{NO}$.

In addition to CSCR and NO, abnormally increased SFCT has been previously reported in many different conditions in inflammatory conditions (e.g Vogt-KoyanagiHarada). Agrawal et al. [18]. published results regarding patients with VKH disease and compared SFCT and CVI with a healthy control group. Both parameters were significantly higher at baseline than follow-up, and also between VKH eyes and controls. They hypothesized that there may be an increased vascular component in VKH eyes compared to normal eyes. Both diseases, CSCR and VKH cause increased choroidal thickness but CVI was also increased in these patients because of the increased vascular component. In this study, we found that vascular and stromal component were both increased, thus CVI was not changed in $\mathrm{NO}$ eyes.

In conclusion, as a novel OCT-based marker, CVI could be used to assess vascular status of the choroid in eyes with $\mathrm{NO}$ and this measurement can provide better understanding of the pathogenesis of this disease.

\section{Summary}

\section{What was known before}

- Eyes with nanophthalmos have increased choroidal thickness.

- Choroidal vascularity index is increased in several diseases, such as CSCR and posterior uveitis.

\section{What this study adds}

- Choroidal vascularity index was not changed in nanophthalmos eyes, however there is a proportional increase in the vascular and stromal component of choroid.

- This novel OCT-based marker can provide better understanding of the pathogenesis of this disease.

\section{Compliance with ethical standards}

Conflict of interest The authors declare that they have no conflict of interest.

Publisher's note Springer Nature remains neutral with regard to jurisdictional claims in published maps and institutional affiliations.

\section{References}

1. Duke-Elder S. Normal and abnormal development. Congenital deformities. In: Duke-Elder S, editor. System of opthalmology, vol. 3. St Louis, MO: CV Mosby; 1964. p. 488-95.

2. Areiter E, Neale M, Johnson SM. Spectrum of angle closure, uveal effusion syndrome, and nanophthalmos. J Curr Glaucoma Pr. 2016;10:113-7.

3. Kimbrough RL, Trempe CL, Brockhurst RJ. Angle-closure glaucoma in nanophthalmos. Am J Ophthalmol. 1979;88:572.

4. Kara N, Baz O, Altinkaynak H, Altan C, Demirok A. Assessment of the anterior chamber angle in patients with nanophthalmos: an anterior segment optical coherence tomography study. Curr Eye Res. 2013;38:563-8.

5. Altan C, Kara N, Baz O, Satana B, Demirok A, Yilmaz OF. Corneal biomechanical properties and intraocular pressure measurement in patients with nanophthalmos. Br J Ophthalmol. 2012;96:806-10.

6. Brockhurst RJ. Nanophthalmos with uveal effusion: a new clinical entity. Arch Ophthalmol 1975;93:1289-99.

7. Mansour A, Stewart MW, Shields CL, Hamam R, Fattah MA, Sheheitli $\mathrm{H}$, et al. Extensive circumferential partial-thickness sclerectomy in eyes with extreme nanophthalmos and spontaneous uveal effusion. Br J Ophthalmol. 2019;103:1862-7.

8. Agrawal R, Gupta P, Tan KA, Cheung CMG, Wong TY, Cheng $\mathrm{CY}$. Choroidal vascularity index as a measure of vascular status of the choroid: measurements in healthy eyes from a populationbased study. Sci Rep. 2016;6:21090.

9. Sonoda S, Sakamoto T, Yamashita T, Uchino E, Kawano H, Yoshihara N, et al. Luminal and stromal areas of choroid determined by binarization method of optical coherence tomographic images. Am J Ophthalmol. 2015;159:1123-31.e1.

10. Agrawal R, Chhablani J, Tan KA, Shah S, Sarvaiya C, Banker A. Choroidal vascularity index in central serious chorioretinopathy. Retina. 2016;36:1646-51.

11. Rizzo S, Savastano A, Finocchio L, Savastano MC, Khandelwal N, Agrawal R. Choroidal vascularity index changes after vitreomacular surgery. Acta Ophthalmol. 2018;96:e950-5.

12. Sonoda S, Sakamoto T, Yamashita T, Shirasawa M, Uchino E, Terasaki $\mathrm{H}$, et al. Choroidal structure in normal eyes and after photodynamic therapy determined by binarization of optical coherence tomographic images. Invest Ophthalmol Vis Sci. 2014;55:3893-9.

13. Zhang Z, Zhang S, Jiang X, Wei Y. Combined 23-G pars plana vitrectomy and lensectomy in the management of glaucoma associated with nanophthalmos. Ophthalmic Res 2018;59:37-44.

14. Demircan A, Altan C, Osmanbasoglu OA, Celik U, Kara N, Demirok A. Subfoveal choroidal thickness measurements with enhanced depth imaging optical coherence tomography in patients with nanophthalmos. Br J Ophthalmol. 2014;98:345-9.

15. Kumar V, Azad SV, Vohra R, Venkatesh P. Serous macular detachment in nanophthalmos: a manifestation of pachychoroid spectrum. Am J Ophthalmol Case Rep. 2019;15:100522. 
16. Summers JA. The choroid as a sclera growth regulator. Exp Eye Res. 2013;114:120-7.

17. Marzani D, Wallman J. Growth of the two layers of the chick sclera is modulated reciprocally by visual conditions. Invest Ophthalmol Vis Sci. 1997;38:1726-39.
18. Agrawal R, Li LKH, Nakhate V, Khandelwal N, Mahendradas P. Choroidal vascularity index in Vogt-Koyanagi-Harada disease: an EDI-OCT derived tool for monitoring disease progression. Transl Vis Sci Technol. 2016;5:7. 